\title{
Anemia no Brasil: até quando?
}

\author{
Anemia in Brazil: how long?
}

$\mathrm{O}$ artigo publicado neste número ${ }^{1}$ refere-se a estudo realizado na região semi-árida do Estado de Alagoas, em que metade das gestantes avaliadas apresentou anemia.

Apesar de se utilizar o termo "anemia" como sinônimo de deficiência de ferro, não se pode ignorar que determinações que consideram apenas os níveis de hemoglobina inferiores a $11 \mathrm{~g} / \mathrm{dL}$ devem ser corretamente denominadas de "anemia, somente.

Entretanto, os resultados deste estudo, com relação aos fatores socioeconômicos desfavoráveis associados à anemia, sugerem que, pelo menos nessa população, a deficiência de ferro é a potencial causa da anemia entre as gestantes. Altos índices de anemia são encontrados em outras regiões do Brasil, como demonstrado em revisão recente ${ }^{2}$, em que se incluíram pesquisas das últimas três décadas em crianças, mulheres em idade fértil e gestantes. Essa revisão concluiu que houve aumento dessa prevalência ao longo do tempo, com leve tendência de diminuição nas publicações que apresentam coleta de dados nos últimos três anos.

Em publicação recente da Organização Mundial de Saúde (OMS) ${ }^{3}$, que envolveu a compilação de dados de 93 países de 1993 a 2005 foi demonstrado que 1,6 bilhão de pessoas ( $24,8 \%$ da população mundial) são acometidas pela anemia. As maiores proporções foram encontradas na África $(47,5-67,6 \%)$ e o maior número de pessoas no sudeste da Ásia (315 milhões). Nessa publicação da OMS, o Brasil apresentou a anemia como um problema de saúde pública de intensidade moderada e grave para gestantes e pré-escolares, respectivamente, de acordo com os critérios estabelecidos pela $\mathrm{OMS}^{4}$. Estimativas calculadas em uma iniciativa do Fundo das Nações Unidas para a Infância (UNICEF) $)^{5}$ mostraram prevalência de 40,8\% de anemia na América Latina a partir de dados coletados após a década de 1990. No Brasil não dispomos ainda de dados nacionais sobre a prevalência de anemia, mas, em breve, esses valores serão disponibilizados pela Pesquisa Nacional de Demografia e Saúde/2006, a qual realizou a dosagem de hemoglobina em todas as regiões do país entre crianças menores de seis anos e mulheres em idade fértil não grávidas.

A alta prevalência de anemia ferropriva na gestação é decorrente de elevado número de mulheres que já iniciam a gestação com deficiência de ferro associado ao alto requerimento durante a gestação. A justificativa para esse fato é que a mulher em idade fértil é considerada grupo de risco para deficiência de ferro em função da perda menstrual, que corresponde a $0,51 \mathrm{mg}$ de ferro por dia (além da perda basal de $0,8 \mathrm{mg} / \mathrm{dia}$ ), e da ingestão insuficiente desse nutriente. Estima-se que a biodisponibilidade do ferro da alimentação rica em cereais, raízes e tubérculos com negligenciáveis quantidades de carne, peixe ou vitamina $C$ seja de 5

Correspondência:

Márcia Regina Vitolo Rua Sarmento Leite, 245 CEP 90050-170 - Porto Alegre/RS E-mail: vitolo@ufcspa.edu.br
* Professora Adjunta do Departamento de Saúde Coletiva/Curso de Nutrição da Universidade Federal de Ciências da Saúde de Porto Alegre - UFCSPA - Porto Alegre (RS), Brasil. 
a $10 \%{ }^{6}$. Considerando que em cada $1.000 \mathrm{kcal}$ de uma alimentação saudável obtêm-se $6 \mathrm{mg}$ de ferro, a ingestão média recomendada para mulheres adultas de $2.000 \mathrm{kcal}$ fornecerá $12 \mathrm{mg}$ de ferro. Com essa ingestão aliada à biodisponibilidade de $5 \%$, a absorção será de $0,6 \mathrm{mg}$, valor abaixo do recomendado o qual é de 1,36 mg diariamente para uma mulher adulta. Dessa forma, esse balanço negativo vai esgotar as reservas de ferro da mulher, a qual deve ser garantida por meio de alimentação que forneça ferro de alta biodisponibilidade e alimentos enriquecidos. Esses cálculos ajudam a justificar, em parte, as bases da alta prevalência de anemia na gestação. Durante esse período é necessário que a mulher tenha $500 \mathrm{mg}$ de reserva de ferro para manter o adequado balanço hematopoiético ${ }^{7}$. O adicional energético do período gestacional de $300 \mathrm{kcal}$ não é suficiente para aumentar significativamente o aporte de ferro, além do requerimento diário elevado de $27 \mathrm{mg}$ de ferro ${ }^{8}$ que não é possível ser obtido por meio da alimentação básica. Pesquisa recente ${ }^{9}$ no Paquistão, com 1.369 gestantes entre 20 e 26 semanas gestacionais mostrou alta prevalência de anemia e associação com o consumo de carne menor que três vezes por semana.

Com relação à prática da suplementação, recomenda-se para todas as gestantes a suplementação de 30 a $40 \mathrm{mg}$ no último trimestre como medida profilática ${ }^{4}$ para garantir a manutenção das reservas de ferro e suprir os requerimentos gestacionais. Na presença de anemia ou deficiência de ferro, recomenda-se suplementação de 60 a 80 mg. No entanto, devem-se evitar suplementações com doses maiores do que essas, em função da possibilidade de se aumentar o requerimento de zinco e levar à deficiência desse nutriente na presença de ingestões marginais. Além do mais, suplementações medicamentosas excessivas acentuam os efeitos colaterais ${ }^{10}$, diminuindo a adesão ao tratamento que constitui umas das causas principais do fracasso da efetividade da suplementação de ferro. Essa limitação é observada na infância e gestação. Pesquisadores da Tanzânia fazem críticas ao Ministério de Saúde do país quanto à falha em disponibilizar, de forma sistemática, os suplementos de ferro e folato para gestantes e, portanto defendem o uso de suplemento alimentar enriquecido com ferro no lugar de suplementos medicamentosos ${ }^{11}$.

No Brasil, as farinhas de milho e trigo são fortificadas com ferro desde 2004, sendo que, a cada $100 \mathrm{~g}$ de farinha, obtêm-se $4 \mathrm{mg}$ de ferro. Nessa proporção, seriam necessários cinco pães franceses, ou a mesma quantidade de outro alimento equivalente para fornecer um terço da quantidade diária de 12 a $13 \mathrm{mg}$, as quais são recomendadas na alimentação saudável para mulheres adultas. Entretanto, para gestantes essa quantidade equivaleria a um sétimo, aproximadamente, constituindo-se numa medida fraca para colaborar com a ingestão adequada de ferro.

Diante desse panorama, quais são os prejuízos associados à anemia? Além das conseqüências bem conhecidas, como o retardo de crescimento intra-uterino, baixo peso ao nascer, prematuridade, crianças com reserva de ferro depletada e insuficiência cardíaca materna, estudos recentes têm considerado também o maior risco de contaminação por chumbo quando há anemia na gestação ${ }^{12}$ e prejuízos na função da tireóide ${ }^{13}$. A análise do ranking das principais causas de anos de vida perdidos por morte prematura e/ou por incapacidade (disability adjusted life years, DALY) mostrou que a deficiência de ferro ocupou o nono lugar entre as 20 causas principais ${ }^{14}$. $\mathrm{Na}$ África, $81 \%$ do total de DALY são originários da mortalidade associada à anemia na gestação. Na América Latina, esse número não é tão mais baixo correspondendo a $61 \%$. Por outro lado na América do Norte e Cuba esse número é de 10\% do total de DALY. Considerando que esses países apresentam baixas taxas de mortalidade, o restante da carga da doença é atribuído às seqüelas diretas como prejuízos cognitivos e na força de trabalho ${ }^{15,16}$. Uma metanálise mostrou que há diminuição do risco de mortalidade materna e perinatal para cada $1 \mathrm{~g} / \mathrm{dL}$ de aumento na média da concentração de hemoglobina durante a gestação ${ }^{15}$.

Essa situação nos instiga a perguntar por quanto tempo serão realizados estudos de avaliação sem que se priorizem investigações, as quais busquem soluções efetivas de combate a essa condição que há três décadas se mantém altamente prevalente em todo país. É possível que a suplementação medicamentosa seja efetiva, mas para isso, é necessária prescrição sistemática, com distribuição gratuita nas unidades básicas de saúde e nível de escolaridade suficiente $^{17}$, um requisito fundamental para que orientações e informações difundidas tanto no âmbito do serviço de saúde quanto nos canais informais de comunicação possam influenciar a adesão ao tratamento. Não é possível ignorar, entretanto, fatores culturais no processo de mudanças nas práticas alimentares, além das limitações socioeconômicas, já que o consumo de vísceras uma vez por semana constitui-se em prática alimentar que fornece ferro de alta biodisponibilidade e de baixo custo. A Coordenação da Política Nacional de Alimentação e Nutrição do Ministério da Saúde incluiu como diretriz do Guia Alimentar para a população brasileira, a inclusão desses alimentos como medida preventiva da deficiência de micronutrientes ${ }^{18}$. Ressalta-se também que a deficiência de outros micronutrientes, como folato e vitamina B12, pode estar fortemente associada à anemia e que, dependendo da condição nutricional da gestante eles devem fazer parte da rotina da suplementação ${ }^{19-21}$.

Assim, aproveitamos esse espaço de reflexão para sugerir aos órgãos de fomento federais ou estaduais, que direcionem os investimentos em pesquisa para a elaboração de programas que combatam a anemia entre os grupos populacionais vulneráveis e que, a partir de resultados efetivos, esses programas possam ser implementados de 
forma sistemática para que novas pesquisas de avaliações possam ser realizadas, com o intuito de conhecer o novo panorama que se instituirá após medidas preventivas. Caso contrário continuará se medindo as dimensões de um problema do qual já são conhecidos os resultados. O estudo nesta edição acrescenta que as variáveis vinculadas à pobreza e às condições biológicas insatisfatórias estão fortemente associadas à presença da anemia entre gestantes e que o desafio, portanto, vai além do âmbito da Saúde.

\section{Referências}

1. Ferreira HS, Moura FA, Cabral Junior CR. Prevalência e fatores associados à anemia em gestantes da região semi-árida do estado de Alagoas, Brasil. Rev Bras Ginecol Obstet. 2008; 30(9): 445-51.

2. Batista Filho M, Souza Al, Miglioli TC, Santos MC. Anemia e obesidade: um paradoxo da transição nutricional brasileira. Cad Saude Publica. 2008;24(supl.2):s247-s257.

3. Benoist B, McLean E, Egli I, Cogswell M, editors. Worldwide prevalence of anaemia 1993-1995: WHO Global Database on Anaemia. Geneva: WHO; 2008

4. World Health Organization. Iron deficiency anaemia: assessment, prevention, and control. A guide for programme managers [WHO/ NHD/01.3]. Geneva: World Health Organization; 2001.

5. Mason J, Rivers J, Helwig C. Recent trends in malnutrition in developing regions: vitamin A deficiency, anemia, iodine deficiency, and child underweight. Food Nutr Bull. 2005;26(1):57.

6. World Health Organization. Vitamin and mineral requirements in human nutrition. 2nd ed. Geneva: WHO; 2004.

7. Schümann K, Ettle T, Szegner B, Elsenhans B, Solomons NW. On risks and benefits of iron supplementation recommendations for iron intake revisited. J Trace Elem Med Biol. 2007;21(3):147-68.

8. US Institute of Medicine, Food and Nutrition Board, Standing Committee on the Scientific Evaluation of Dietary Reference Intakes. Dietary reference intakes for vitamin A, vitamin K, Arsenic, Boron, Chromium, Copper, lodine, Iron, Manganese, Molybdenum, Nickel, Silicon, Vanadium, and Zinc. Washington, D.C.: National Academy Press; 2001

9. Baig-Ansari N, Badruddin SH, Karmaliani R, Harris H, Jehan I, Pasha O, et al. Anemia prevalence and risk factors in pregnant women in an urban area of Pakistan. Food Nutr Bull. 2008;29(2):132-9

10. Rioux FM, LeBlanc CP. Iron supplementation during pregnancy: what are the risks and benefits of current practices? Appl Physiol Nutr Metab. $2007 ; 32(2): 282-8$.

11. Latham MC, Ash DM, Makola D, Tatala SR, Ndossi GD, Mehansho H. Efficacy trials of a micronutrient dietary supplement in schoolchildren and pregnant women in Tanzania. Food Nutr Bull. 2003;24(4 Suppl):S120-8.

12. Zentner LE, Rondó PH, Duran MC, Oliveira JM. Relationships of blood lead to calcium, iron, and vitamin C intakes in Brazilian pregnant women. Clin Nutr. 2008;27(1):100-4.

13. Zimmermann MB, Burgi H, Hurrell RF. Iron deficiency predicts poor maternal thyroid status during pregnancy. J Clin Endocrinol Metab. 2007;92(9):3436-40.

14. Ezzati M, Lopez AD, Rodgers A, Vander Hoorn S, Murray CJ; Comparative Risk Assessment Collaborating Group. Selected major risk factors and global and regional burden of disease. Lancet. 2002;360(9343):1347-60.

15. Stoltzfus RJ. Iron deficiency: global prevalence and consequences. Food Nutr Bull. 2003;24(4 Suppl):S99-S103.

16. Schramm JMA, Oliveira AF, Leite IC, Valente JG, Gadelha AM, Portela MC, et al. Transição epidemiológica e o estudo de carga de doença no Brasil. Cienc Saude Coletiva. 2004;9(4):897-908.

17. Vitolo MR, Boscaini C, Bortolini GA. Baixa escolaridade como fator limitante para o combate à anemia entre gestantes. Rev Bras Ginecol Obstet. 2006;28(6):331-9.

18. Brasil. Ministério da Saúde. Secretária de Atenção a Saúde. Departamento de Atenção Básica. Coordenação-Geral da Política de Alimentação e Nutrição. Guia alimentar da população brasileira: promovendo a alimentação saudável. Brasília, DF: Ministério da Saúde; 2005.

19. Kontic-Vucinic O, Sulovic N, Radunovic N. Micronutrients in women's reproductive health: II. Minerals and trace elements. Int J Fertil Womens Med. 2006;51(3):116-24.

20. Kontic-Vucinic O, Sulovic N, Radunovic N. Micronutrients in women's reproductive health: I. Vitamins. Int J Fertil Womens Med. 2006;51(3):106-15.

21. Vanderjagt DJ, Brock HS, Melah GS, El-Nafaty AU, Crossey M, Glew RH. Nutritional factors associated with anaemia in pregnant women in northern Nigeria. J Health Popul Nutr. 2007;25(1):75-81. 